\title{
Cost-Effectiveness of an Obesity Management Program for 6- to 15-Year-Old Children in Poland: Data from Over Three Thousand Participants
}

\author{
Ewa Bandurska ${ }^{a}$ Michał Brzeziński ${ }^{b, c}$ Paulina Metelska ${ }^{a}$ b Marzena Zarzeczna-Baran ${ }^{a}$ \\ ${ }^{a}$ Department of Public Health and Social Medicine, Medical University of Gdansk, \\ Gdansk, Poland; ${ }^{b}$ 6-10-14 for Health Program, University Clinical Center in Gdansk, \\ Gdansk, Poland; ${ }^{c}$ Department of Paediatrics, Gastroenterology, Allergology and \\ Paediatric Nutrition, Gdansk, Poland
}

\section{Keywords}

Pediatric obesity · Behavioral intervention · Interdisciplinary intervention · Obesity

management program

\begin{abstract}
Background: Obesity and overweight, including childhood obesity and overweight, pose a public health challenge worldwide. According to the available research findings, long-term interventions focusing on dietary behavior, physical activity, and psychological support are the most effective in reducing obesity in children aged 6-18 years. There are limited studies showing the financial effectiveness of such interventions. Objective: The objective of the present study was to evaluate cost-effectiveness of the 6-10-14 for Health weight management program using pharmacoeconomic indicators, i.e., cost-effectiveness analysis using the incremental cost-effectiveness ratio. Methods: We used anthropometric data of 3,081 children included in a 1-year-long intervention with a full financial cost assessment. Results: The cost of removing a child from the overweight group (BMI > 85th percentile) was PLN 27,758 (EUR 6,463), and the cost of removing a child from the obese group (BMI >95th percentile) was slightly lower, i.e., PLN 23,601 (EUR 5,495). Given the obesity-related medical costs calculated in the life-long perspective, these results can be considered encouraging. At the same time, when comparing the total costs per participant with the costs of other interventions, it can be noted that they are similar to the costs of school programs containing more than 1 type of intervention. Conclusions: The 6-10-14 for Health program can be considered cost-effective. As a result of committing financial resources in the approximate amount of EUR 1,790 per child, around half of the children participating in the program have improved their weight indicators.
\end{abstract}


Bandurska et al.: Multidisciplinary Obesity Management Programs for Children Can Be Cost-Effective

\section{Introduction}

\section{Background}

Obesity and overweight, including childhood obesity and overweight, pose a public health challenge worldwide [1-3]. This disease increases the risk of diabetes, hypertension, disability, and premature death. Overweight and obese children are at a greater risk for becoming overweight or obese adults and developing noncommunicable diseases $[4,5]$. It is estimated that 41 million children under the age of 5 years are obese and 340 million children in the 5- to 19-year-old age group are affected by obesity [2]. A similarly unfavorable situation occurs in Poland. According to the available national data, the prevalence of overweight and obesity in children and adolescents aged $7-18$ years is $18.8-24.6 \%$ in boys and $14.3-17.4 \%$ in girls [6]. In view of the increasing prevalence rates of obesity and overweight, including severe obesity in children [3], it is considered necessary to prevent them using tools with proven effectiveness. The available publications indicate that programs targeting children and young people to promote physical activity and diet-changing interventions in both children and their families are the most likely to bring about sustainable lifestyle changes [7]. As obesity and childhood overweight are a complex problem with many social and environmental factors, the solutions must be multidimensional, tailored to the context and individual needs of the actors involved, and maintained throughout the life of the child [8]. According to the available research findings, systematic reviews, and meta-analyses, long-term interventions focusing on dietary behavior, physical activity, and psychological support are the most effective in reducing obesity in children aged 6-18 years $[9,10]$. These programs should be implemented by interdisciplinary teams of specialists [11]. These criteria are met by the 6-10-14 for Health program running in Gdansk since 2011. The program covers children aged 6-14 years who, after a screening examination, are enrolled into a 12-month comprehensive health intervention consisting of a program of 4 multidisciplinary consultations (medical, nutrition-related, psychological, and regarding physical activity) carried out in a cycle of 0-3-6-12 months. The visits are always attended by a child and at least one parent/guardian. A detailed description of the program is available in previous publications $[12,13]$.

\section{Objective}

The purpose of this study is to evaluate the cost-effectiveness of an integrated and individually run program for children aged 6-14 through:

- assessment of weight-related clinical indicators, including BMI, BMI percentile, $z$-score, and body fat percentage (BFP);

- evaluation of the costs of the program; and

- evaluation of pharmacoeconomic indicators, i.e., cost-effectiveness analysis (CEA) using an incremental cost-effectiveness ratio (ICER).

\section{Materials and Methods}

\section{Intervention}

The program is an individual, integrated program the main objective of which is to reduce the prevalence of obesity and overweight in the population of schoolchildren and adolescents. The program includes a number of comprehensive modules, such as:

1. screening by educational professionals to identify the population of children and adolescents at high risk for civilization diseases, i.e., overweight and obesity defined according to the BMI percentiles identified in the OLAF (a representative Polish population study) [14]; 
2. specialist care for children with excess body weight, which includes visits to a pediatrician, a physical activity specialist, a nutritionist, and a psychologist, with each intervention involving, apart from the child, at least one parent/guardian;

3. parental education, i.e., educational workshops for parents and guardians and for participants in the program aged over 14 years; and

4. specialist consultations, i.e., additional visits to specialists, for example, to an endocrinologist, if the pediatrician identifies such a need.

The full cycle of the program includes 4 meetings in which both the children and their guardians participate. The meetings are scheduled to take place quarterly and take the form of individual specialist consultations.

There are publications on the effectiveness of obesity prevention programs in children and adolescents, but these are mainly school-, group-, or community-based programs $[9,15$, 16]. The number of publications on individual interventions targeted at children and their relatives is limited [11]. Due to their diversity, different duration, inclusion criteria for the study group, and duration of evaluation, it is very difficult to compare the effects between these programs [15]. In addition, only a few studies have assessed the economic effectiveness of interventions in a pediatric population [17-19].

\section{Analysis of Clinical Indicators}

Body weight and height were measured in the standing position, with the patient in underwear and without shoes, with an accuracy of $0.1 \mathrm{~kg}$ using a digital scale (WE150; Mensor, Poland). The device was calibrated every day. The height was evaluated with an accuracy of $0.001 \mathrm{~m}$. Bioelectrical impedance analysis, including BFP, was evaluated with the use of Tanita BC-420 MA equipment, the correctness of which was verified every 6 months. The BMI $z$-score was estimated on the basis of the following formula:

$$
\mathrm{z} \text {-score }(\mathrm{BMI})=\frac{\mathrm{BMI}-\text { mean BMI }}{\mathrm{SD}}
$$

Body weight, height, and BMI percentile were referenced using the current percentile charts for the Polish population [14].

The main efficacy indicators used were anthropometric indicators such as BMI, BMI percentile, $z$-score (BMI), and BFP. At every measurement, the number of children who achieved a positive change (decrease) in the measured parameters during the observation period was assessed as a positive outcome measure. As a positive change we indicated any reduction in BMI, BMI percentile, or BFP. Additionally, with regard to the BMI measures, the number of children who achieved a decrease of more than 1 percentile and more than 5 percentiles of BMI was determined separately.

Anthropometric parameters were assessed during each visit, quarterly.

\section{Cost Analysis and CEA}

Cost analysis, carried out in the form of a retrospective analysis of the costs of intervention, was performed for the whole period of observation, i.e., for the years 2011-2017. Costs were considered in total and broken down by year, taking into account all costs related to the program by type and including such components as: screening, specialist consultations, equipment, program evaluation, and other costs occurring in the discussed period. The cost amount was estimated on the basis of bills and invoices accepted by the financing institution, i.e., the City Council of Gdansk. 
Bandurska et al.: Multidisciplinary Obesity Management Programs for Children Can Be Cost-Effective

The CEA was carried out by determining the ICER, in which the values of costs and effects of the program were taken into account and compared to a lack of intervention, where the effect and cost were equal to 0 . The ICER was estimated according to the following formula [20]:

$$
\mathrm{ICER}=\frac{C_{1}-C_{2}}{E_{1}-E_{2}}
$$

where $\mathrm{C} 1$ is the cost of a more effective intervention, $\mathrm{C} 2$ is the cost of a less effective intervention, E1 is the effect of a more effective intervention, and E2 is the effect of a less effective intervention

The effect of the intervention (E) in the CEA analysis may take various forms, depending on the type of intervention assessed, but it is always expressed in the form of natural units, i.e., units that are not modified in any way but correspond to what was measured. In the case of weight loss programs, this would be, e.g., the number of kilograms of body weight lost or the average BMI loss (expressed in $\mathrm{kg} / \mathrm{m}^{2}$ ) that was observed in the study population.

\section{Statistical Methods}

All calculations were made using a Microsoft Excel version 2010 spreadsheet and the Statistica statistical package (version 12.5; Statsoft, Poland). The statistical description of quantitative data used classic location measures such as the arithmetic mean and median, as well as SD and range as general measures of variability. The normality of the distribution of continuous variables and the equality of variance of the examined feature in the groups were tested with the Shapiro-Wilk test and the equality of variance test, respectively. When comparing 2 groups for quantitative data, the Student $t$ test was used for parametric data with a normal distribution and homogeneous variances; the Mann-Whitney U test was used in the event of noncompliance with the above assumptions. In order to compare the variability of the examined traits over time, the Student $t$ test for related variables or the Wilcoxon rank test for pair comparisons was used, respectively. Multivariate analysis of variance was used in the analysis of multivariate variability of individual parameters over time. In all statistical tests, $p<0.05$ was considered statistically significant.

\section{Results}

\section{Weight Variables}

The value of weight parameters measured during 4 consecutive visits, which were carried out quarterly, was analyzed. It was shown that the observed changes were mostly statistically significant, with the exception of the change in BMI percentile depending on gender. Parameters were analyzed for all children and broken down by gender and age (younger and older children; Table 1).

Both girls and boys lowered their BMI percentiles during the intervention period. At the last visit, the BMI percentile remained slightly higher in girls than in boys (i.e., 91.3 vs. 89.9) despite similar mean values in both groups during the first visit. The course of changes in BFP was similar, although the value of this parameter stabilized in girls between the 3rd and 4th visits. A similar decrease in BFP occurred in younger and older children between the first 2 visits, but starting from 3th visit the BFP was lower in children from the older age group. Changes in the BMI percentiles were similar in both age groups, i.e., between the 1st and the 3rd visits a dynamic decrease was observed, followed by a further, smaller decrease.

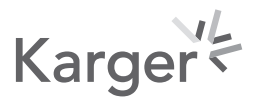


Table 1. Weight parameters obtained during subsequent visits for the whole group, depending on gender and age

\begin{tabular}{|c|c|c|c|c|c|c|c|c|}
\hline & $\begin{array}{l}\text { All children } \\
(n=1,121)\end{array}$ & $p$ value & $\begin{array}{l}\text { Girls } \\
(n=554)\end{array}$ & $\begin{array}{l}\text { Boys } \\
(n=567)\end{array}$ & $p$ value & $\begin{array}{l}\text { Children aged } \\
\leq 10 \text { years } \\
(n=541)\end{array}$ & $\begin{array}{l}\text { Children aged } \\
>10 \text { years } \\
(n=580)\end{array}$ & $p$ value \\
\hline \multicolumn{9}{|c|}{ BMI percentile } \\
\hline \multicolumn{9}{|l|}{ Visit } \\
\hline 1 & $94.44 \pm 3.62$ & 0.00 & $94.66 \pm 3.51$ & $94.22 \pm 3.71$ & 0.03 & $94.68 \pm 3.59$ & $94.21 \pm 3.63$ & 0.54 \\
\hline 2 & $92.62 \pm 5.69$ & & $92.87 \pm 5.83$ & $92.37 \pm 5.56$ & & $92.55 \pm 6.42$ & $92.68 \pm 4.93$ & \\
\hline 3 & $91.22 \pm 7.17$ & & $91.64 \pm 7.27$ & $90.80 \pm 7.05$ & & $91.18 \pm 7.81$ & $91.25 \pm 6.52$ & \\
\hline 4 & $90.55 \pm 8.32$ & & $91.26 \pm 7.84$ & $89.86 \pm 8.72$ & & $90.89 \pm 8.30$ & $90.24 \pm 8.33$ & \\
\hline \multicolumn{9}{|l|}{$B F P$} \\
\hline \multicolumn{9}{|l|}{ Visit } \\
\hline 1 & $29.63 \pm 5.68$ & 0.00 & $32.47 \pm 4.21$ & $26.87 \pm 5.56$ & 0.00 & $29.89 \pm 4.42$ & $29.38 \pm 6.64$ & 0.02 \\
\hline 2 & $28.56 \pm 6.14$ & & $31.68 \pm 5.09$ & $25.49 \pm 5.51$ & & $28.93 \pm 5.39$ & $28.21 \pm 6.75$ & \\
\hline 3 & $27.69 \pm 6.46$ & & $30.98 \pm 4.55$ & $24.43 \pm 6.42$ & & $28.26 \pm 4.92$ & $27.16 \pm 7.58$ & \\
\hline 4 & $27.27 \pm 6.74$ & & $31.13 \pm 4.80$ & $23.5 \pm 6.22$ & & $28.36 \pm 5.35$ & $26.26 \pm 7.69$ & \\
\hline
\end{tabular}

Values are presented as means \pm SD. $p<0.05$ is considered statistically significant (ANOVA).

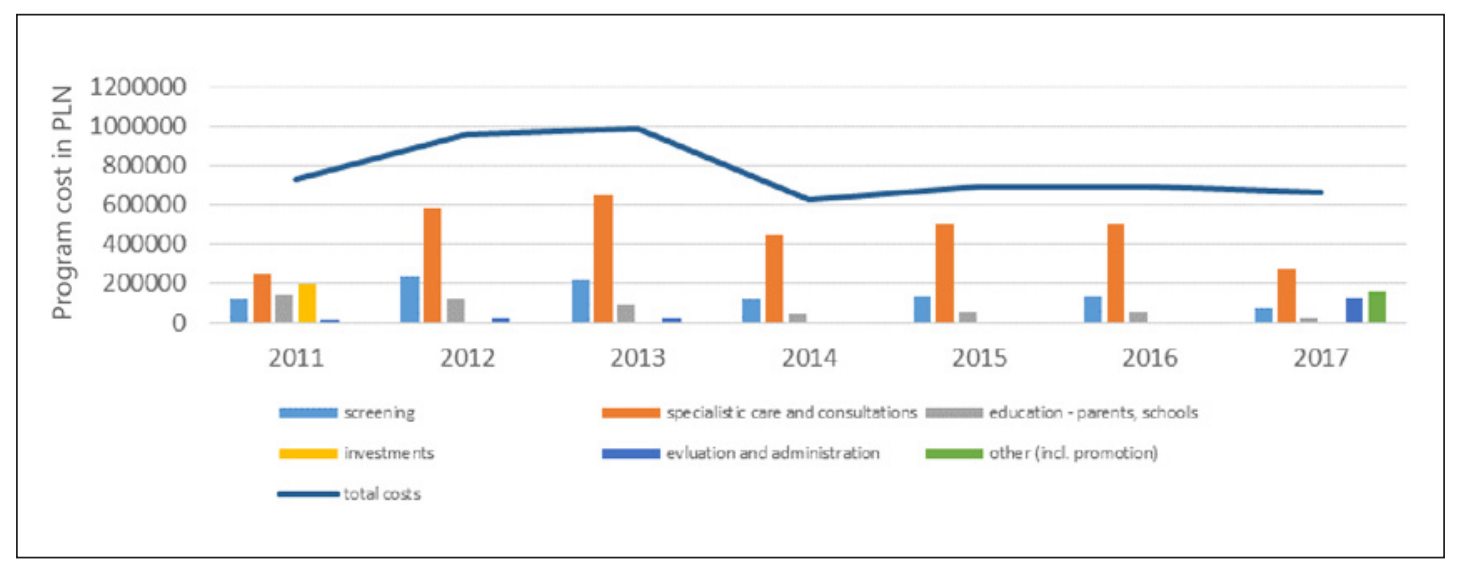

Fig. 1. Program costs by cost type for the years 2011-2017.

CEA: Intervention Costs

All costs are expressed in PLN and EUR (1 EUR $=4.295$ PLN). The average annual costs of the program in 2011-2017 amounted to PLN 765,335 (EUR 169,878), stabilizing at less than PLN 700,000 ( EUR 163,000) from 2014 onwards. The analysis of costs by type showed that the costs of specialist care and other consultations constituted the largest part of the total cost (33.80-72.02\%) (Figure 1).

Costs per capita were calculated separately for all children participating in the program in a given year and for children who had undergone all 4 consultations planned under the program. They amounted on average to PLN 3,087 and PLN 7,689 (EUR 719 and EUR 1,790, respectively) for the duration of the analysis, with the highest amount in the starting year.

CEA: Evaluation of the Program Effect (BMI Percentile)

From the initial group of $100 \%$ of children with a BMI $\geq 85$ percentile, 928 children had a BMI $\geq 85$ percentile at the 4 th visit, which amounted to $82.78 \%$ of the initial group. 
Table 2. Results of the CEA: BMI percentile

\begin{tabular}{|c|c|c|c|c|c|c|c|}
\hline \multirow{2}{*}{$\begin{array}{l}\text { BMI } \\
\text { percentile }\end{array}$} & \multicolumn{7}{|c|}{ Children, $n$} \\
\hline & $\mathrm{v} 1$ & v2 & v3 & $\mathrm{v} 4$ & $\begin{array}{l}\text { falling below } \\
\text { a given } \\
\text { percentile }\end{array}$ & $\begin{array}{l}\text { difference in } \\
\text { number between } \\
\text { v1 and v4 }\end{array}$ & $\begin{array}{l}\text { falling below a } \\
\text { given percentile as } \\
\text { a percent of v1, \% }\end{array}$ \\
\hline$\geq 85$ & 1,121 & 1,025 & 947 & 928 & 193 & -193 & 17.22 \\
\hline$\geq 90$ & 984 & 850 & 762 & 737 & 273 & -247 & 27.74 \\
\hline$\geq 95$ & 624 & 484 & 435 & 442 & 227 & -182 & 36.38 \\
\hline$\geq 97$ & 388 & 292 & 257 & 262 & 159 & -126 & 40.98 \\
\hline$\geq 99$ & 133 & 102 & 87 & 96 & 59 & -37 & 44.36 \\
\hline
\end{tabular}

v, visit.

Table 3. Results of the CEA: other weight variables

\begin{tabular}{ll}
\hline Did the parameter improve? & $\begin{array}{l}\text { Children, } n \\
(\%)\end{array}$ \\
\hline BMI (decrease by at least 0.1) & $623(55.58)$ \\
$\quad$ Yes & $498(44.42)$ \\
$\quad$ No & $540(48.17)$ \\
BMI $z$-score (decrease by at least 0.01) & $581(51.83)$ \\
$\quad$ Yes & $558(49.78)$ \\
$\quad$ No & $563(50.22)$ \\
Fat weight, kg in bioimpedance (decrease by at least 0.1$)$ & \\
$\quad$ Yes & $808(72.08)$ \\
$\quad$ No & $313(27.92)$ \\
Body fat, \% in bioimpedance (decrease by at least 0.01$)$ & \\
Yes & \\
No &
\end{tabular}

The highest number of children $(n=273)$ who moved below their original BMI percentile as measured at the 1st visit were initially in the above-the-90th-percentile group.

The highest percentage of children who left the BMI $\geq 99$ th percentile group (44.36\%) (Table 2).

CEA: Evaluation of the Program Effect (Other Weight Variables)

The other weight parameters also improved in the children included in the program. Improvements in BMI (decrease by at least 0.1 ), $z$-score (decrease by at least 0.01 ), and bioimpedance of body fat (in kg; decrease by at least 0.1 ) were observed in more than half of the participants, while a decrease in BFP was seen in nearly 3 quarters (Table 3 ).

\section{Cost-Effectiveness Analysis}

The CEA showed that the cost of improving a weight parameter was the lowest when reducing body fat (EUR 1,544), followed by a BMI percentile drop of at least 1 percentile. The lowest cost-effectiveness was found for children leaving the percentile group >99 (EUR 21,141 ), but this was also the group with the most serious health problems (Table 4).

The cost of removing a child from the overweight group (BMI >85th percentile) was PLN 27,758 (EUR 6,463); the cost of removing a child from the obesity group (BMI >95th percentile) was slightly lower, PLN 23, 601 (EUR 5,495). 
Bandurska et al.: Multidisciplinary Obesity Management Programs for Children Can Be Cost-Effective

Table 4. ICER results compared to no intervention

\begin{tabular}{lcc}
\hline Parameter & \multicolumn{2}{l}{ ICER compared to no intervention } \\
\cline { 2 - 2 } & PLN & EUR \\
\hline BMI percentile - different variants & & \\
Child's exit from >85th percentile & 27,758 \\
Child's exit from >90th percentile & 19,624 & 4,569 \\
Child's exit from >95th percentile & 23,601 & 5,495 \\
Child's exit from >97th percentile & 33,694 & 7,845 \\
Child's exit from >99th percentile & 90,803 & 21,141 \\
Decrease >1 BMI percentile & 6,949 & 1,618 \\
Decrease $>5$ BMI percentiles & 7,115 & 1,657 \\
Other weight parameters & & 1,964 \\
BMI (change by at least 0.1) & 8,437 & 2,310 \\
Z-score ( change by at least 0.01 ) & 9,921 & 2,200 \\
Body fat (kg; change by at least 0.1$)$ & 9,449 & 1,544 \\
BFP (change by at least 0.01) & 6,630 & \\
\hline
\end{tabular}

Table 5. Social cost savings depending on the percentage of participants with a BMI $>95$ th percentile reducing their body mass

\begin{tabular}{rrrrrrr}
\hline $\begin{array}{l}\text { Children who exited } \\
\text { 95 percentile }\end{array}$ & $\begin{array}{l}\text { 1-year } \\
\text { savings, PLN }\end{array}$ & $\begin{array}{l}\text { 1-year } \\
\text { savings, } \\
\text { EUR }\end{array}$ & $\begin{array}{l}\text { 10-year } \\
\text { savings, PLN }\end{array}$ & $\begin{array}{l}\text { 10-year } \\
\text { savings, } \\
\text { EUR }\end{array}$ & $\begin{array}{l}\text { Life expectancy } \\
\text { (67.15 years) } \\
\text { savings, PLN }\end{array}$ & $\begin{array}{l}\text { Life expectancy } \\
\text { (67.15 years) } \\
\text { savings, EUR }\end{array}$ \\
\hline 100 & $4,061,121$ & 945,546 & $40,611,210$ & $9,455,462$ & $272,704,275$ & $63,493,428$ \\
75 & $3,045,841$ & 709,160 & $30,458,410$ & $7,091,597$ & $204,528,223$ & $47,620,075$ \\
50 & $2,030,560$ & 472,773 & $20,305,600$ & $4,727,730$ & $136,352,104$ & $31,746,706$ \\
25 & $1,015,280$ & 236,386 & $10,152,800$ & $2,363,865$ & $68,176,052$ & $15,873,353$ \\
10 & 547,020 & 127,362 & $\mathbf{5 , 4 7 0 , 2 0 0 *}$ & $\mathbf{1 , 2 7 3 , 6 2 0 *}$ & $36,732,393$ & $8,552,362$ \\
5 & 203,056 & 47,277 & $2,030,560$ & 472,773 & $13,635,210$ & $3,174,671$ \\
1 & 40,613 & 9,456 & 406,130 & 94,559 & $2,727,163$ & 634,962 \\
\hline
\end{tabular}

* Profitability threshold (details in text).

\section{Long-Term Simulation Model}

There is a significant lack of data on the clinical and cost-effectiveness of similar programs. To assess long-term savings due to reduced social costs of obesity, we performed a microsimulation on all children who decreased their body mass below the 95th centile (starting from above the 95th). We used a microsimulation model that showed that the yearly cost of obesity lasting a minimum of 10 years is 4,280 USD (17,890 PLN)/person [21] (Table 5).

Assuming that the total cost of the program is 5,357,351.48 PLN $(1,247,346$ EUR), it is impossible to earn a net profit even if $100 \%$ of children $(n=227)$ keep their body mass on a starting (decreased) level. In this situation the profitability threshold is gained after 16 months.

In the case of a 10-year horizon (according to a microsimulation study by Su et al. [21]) the profitability threshold is a number of $10 \%$ of the participants who would be able to keep their BMI centile levels. In case of analysis of the data with reference to the expected life expectancy (i.e., 65.17 years for an 11-year-old child, as a medium for the population studied in relation to age and sex) the profitability threshold is equal to maintenance of the BMI parameters by 5 people from the whole group. 
Bandurska et al.: Multidisciplinary Obesity Management Programs for Children Can Be Cost-Effective

\section{Discussion}

Developing and running a multidisciplinary program for the management of pediatric obesity can be a clinically and economically effective intervention. Significant change in anthropometric measures can be achieved with a reasonable financial investment, comparable to other population-based interventions targeted at children and their families living with overweight and obesity.

The subject matter of evaluation of the effectiveness of intervention programs in obese and overweight children is quite popular. However, while there is a clear consensus on the methods for evaluation of clinical effectiveness (i.e., the type of indicators used and the methods for their evaluation), in terms of the cost-effectiveness of the population (interventional studies) there is a lack of a specific model to apply, recommendations, or standard research methodology in general. When analyzing previously published studies assessing cost-effectiveness, it becomes clear that the most frequently used analytical method is CEA, where the cost analysis includes the costs of the intervention, and the analyzed clinical effectiveness factors include weighted effect coefficients [22]. These assumptions were used in the evaluation of the 6-10-14 for Health program. It is difficult to compare the program directly with other interventions related to childhood obesity due to its unique design, i.e., a multidisciplinary approach (specialist care and education) involving individual families.

In most programs, the intervention consists of education aimed at changing lifestyles and is addressed to the child or the whole family. The impact on weight indicators varies according to the type of intervention, but the results speak in favor of lifestyle-changing interventions rather than pharmacological ones (change in BMI or BMI $z$-score of -0.53 vs. -0.43 ) and individually targeted interventions ( -0.90 vs. -0.34 ) [23], but with interventions targeted at specific families having a more positive impact. In the case of this type of intervention, the estimated lifelong benefits are also the greatest $[17,24]$.

A significant proportion of obesity intervention programs are carried out in schools [10], where health education is included in the curriculum; some of the interventions also include increasing the number of hours of PE classes [25]. A study by Moodie et al. [26], investigated the effectiveness of physical activation measures to reduce BMI in a group of younger and older children ( $>10$ years of age). Better results were achieved by older children $(-0.08)$ than by younger children $(-0.07)$. In this study the children achieved a -0.3 average decrease in their median BMI, while, as in the study cited above, older children achieved a better result $(-0.9$ vs. -0.1$)[27,28]$. However, the effectiveness of school programs is questionable [27], and in comparison with the 6-10-14 for Health program it is noticeably lower, which has also been confirmed in studies by other authors. For example, a 2015 meta-analysis by Peirson et al. [23] showed that only interventions involving both dietary and activation interventions can be considered effective (BMI reduction of -0.09 , i.e., less than in this study). Similarly, a meta-analysis by Wang et al. [29] showed that school-based interventions contributed to improvement in any of the weight measurements in children in $22-67 \%$ of cases. The 2017 study, which included annual support interventions in the child's environment, showed statistically significant improvements only in the BMI $z$-score, but changes in body structure (body fat and muscle mass percentages) were similar to those in the control group [30]. Nevertheless, the researchers considered the intervention to be effective in terms of the health impact.

In the 6-10-14 for Health program, an improvement in BFP was achieved in nearly 3 quarters $(72.08 \%)$ of the children, and about half of them achieved an improvement in other parameters, which indicates a high effectiveness of the program. On the other hand, an RCT, which analyzed the effectiveness of a program aimed at strengthening parental competence in order to improve the lifestyle of the whole family, showed a lack of clinical and cost effec-

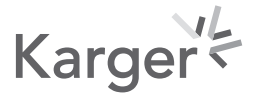


Bandurska et al.: Multidisciplinary Obesity Management Programs for Children Can Be Cost-Effective

tiveness. Robertson et al. [31] demonstrated that not only did the weight parameters of the children from the study group not significantly improve in relation to the control group but in some of them the indicators deteriorated. For example, the average BFP of $36.81 \%$ at the starting point reached $37.58 \%$ after 12 months of the program. In the $6-10-14$ for Health program, a decrease of $-2.36 \%$ in the mean value was noted.

The cost analysis showed that in the 6-10-14 for Health program the calculated cost per capita for all children was EUR 719 and, taking into account only those children who had completed all visits, it was EUR 1,790. Given the obesity-related medical costs calculated in the life-long perspective, these results can be considered encouraging [32, 33]. At the same time, when comparing the total costs per participant with the costs of other interventions, it can be noted that they are similar to the costs of school programs containing more than 1 type of intervention. The advantage of the 6-10-14 for Health program consists of its multidimensional character, the involvement of the child's whole social environment in the intervention, and support from specialists at each stage of the program's implementation.

In a study by Wang et al. [34] describing a school program containing activation components, introducing dietary changes with regard to healthy snacks, and school support (e.g., in education), the cost per capita was USD 558 for all children, respectively, and for children who participated in at least $40 \%$ of activities it was USD 956. Achieving a positive effect in the form of a reduction of the BFP cost an additional USD 317 per capita (a total of USD 1,273).

The costs of obtaining a positive clinical effect, expressed with the use of the abovementioned indicators, were also compared. In a study conducted in 2016, which included an intervention carried out by a multidisciplinary team mainly in a children's home, the cost of improving the BMI $z$-score parameter was USD 1,643 (EUR 1,964 in the 6-10-14 for Health program) and the cost per capita was USD 1,813 [35]. However, only 1 indicator was used in this study, which makes it impossible to carry out a comparison with other performance indicators presented for the 6-10-14 for Health program.

As we have shown, basing on a microsimulation, the population effects of such interventions can be seen in a 10 -year-long perspective and only $5 \%$ of all participants will be able to sustain changes in body composition throughout that period. Unfortunately, there is a very limited pool of data showing whether this effect is possible in population interventions [11, 36].

\section{Strengths and Limitations}

Given the wide variety of parameters used to analyze the effectiveness of antiobesity programs in children, further analysis is necessary. It is also a limitation of this study that it was difficult to conduct a meaningful comparative analysis of cost-effectiveness. While there is a large number of studies on clinical efficacy, there is a lack of consistency in economic analysis in terms of methodology.

The strengths of this work are the large study group (more than 1,100 children who completed a full annual cycle of activities) and the use of various clinical efficacy indicators, which were subsequently used to determine the cost-effectiveness of the program. We can also firmly state that the presented model was positively assessed by the Polish Health Technology Assessment Agency (AOTMiT) and it has been introduced in original or similar form in several other cities in Poland (Warszawa, Szczecin, and Lublin) [37].

We need to acknowledge several possible limitations of the program that could have influenced final results:

- we cannot directly connect any costs with direct patients as the program is mainly financed on indirect costs and medical personnel costs;

- the high dropout rate of patients may have influenced the final results of the program and both the clinical and the economic analyses; to our best knowledge and accotrding 
Bandurska et al.: Multidisciplinary Obesity Management Programs for Children Can Be Cost-Effective

to the data analysis we did not see any specific characteristics of patients not engaging in the next stages of the program; we found that the group in the first and last visits was similar in terms of family composition, income, parents' education level, and readiness to change, yet we cannot exclude a potential bias due to a higher resignation rate of patients with a smaller clinical change; and

- the structure and organisation of the program is mostly suitable for medium/big cities with access to well-trained and motivated specialists as well as a screening infrastructure; Acting in more rural, less urbanized area would require some changes in the program protocol, e.g., screening by school nurses or larger GP office engagement in the preliminary assessment and follow-up.

\section{Conclusions}

1. The 6-10-14 for Health program can be considered cost-effective. As a result of committing financial resources in the approximate amount of EUR 1,790 per child, around half of the children participating in the program have improved their weight indicators.

2. The program was most cost-effective with respect to reducing the BFP, followed by achieving a reduction of at least 1 percentile point in the BMI percentile measure.

\section{Acknowledgment}

The authors express their gratitude to all of the participants, their parents, and the members of the 6-10-14 for Health team for their input into this study. The authors would also like to thank the University Clinical Center administrative staff for their help in conducting this study.

\section{Statement of Ethics}

This study was approved by the Local Ethics Committee at the Medical University of Gdansk (decision No. NKBBN/228/2012) on June 25, 2012, in accordance with the requirements of the Helsinki Declaration. Each parent/legal guardian had to express written consent for the child's participation in the program. The 6-10-14 for Health program is retrospectively registered in clinicaltrials.gov (No. NCT04143074).

\section{Conflict of Interest Statement}

The authors declare that they have no competing interests.

\section{Funding Sources}

The 6-10-14 for Health program is fully funded by the City of Gdansk authorities. The City of Gdansk had no financial input into this work or any impact on the design of this study or the collection, analysis, and interpretation of data and the writing of this paper.

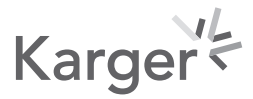




\section{Author Contributions}

Ewa Bandurska and Michal Brzeziński contributed to the study conception and design. Material preparation, data collection, and analysis were performed by Ewa Bandurska, Michal Brzeziński, and Paulina Metelska. The first draft of this paper was written by Ewa Bandurska and Michal Brzeziński, and all of the authors commented on previous versions of this paper. Marzena Zarzeczna-Baran revised the draft of this paper and supervised the work. All of the authors read and approved the final version of this article.

\section{References}

1 Blundell JE, Baker JL, Boyland E, Blaak E, Charzewska J, de Henauw S, et al. Variations in the Prevalence of Obesity Among European Countries, and a Consideration of Possible Causes. Obes Facts. 2017;10(1):25-37.

2 Bentham J, Di Cesare M, Bilano V, Bixby H, Zhou B, Stevens GA, et al.; NCD Risk Factor Collaboration (NCDRisC). Worldwide trends in body-mass index, underweight, overweight, and obesity from 1975 to 2016: a pooled analysis of 2416 population-based measurement studies in 128.9 million children, adolescents, and adults. Lancet. 2017 Dec;390(10113):2627-42.

3 Spinelli A, Buoncristiano M, Kovacs VA, Yngve A, Spiroski I, Obreja G, et al. Prevalence of Severe Obesity among Primary School Children in 21 European Countries. Obes Facts. 2019;12(2):244-58.

4 Farpour-Lambert NJ, Baker JL, Hassapidou M, Holm JC, Nowicka P, O'Malley G, et al. Childhood Obesity Is a Chronic Disease Demanding Specific Health Care-a Position Statement from the Childhood Obesity Task Force (COTF) of the European Association for the Study of Obesity (EASO). Obes Facts. 2015;8(5):342-9.

5 Bjerregaard LG, Jensen BW, Ängquist L, Osler M, Sørensen TI, Baker JL. Change in overweight from childhood to early adulthood and risk of type 2 diabetes. N Engl J Med. 2018 Apr;378(14):1302-12.

6 Kułaga Z, Grajda A, Gurzkowska B, Wojtyło M, Góźdź M, Litwin M. The prevalence of overweight and obesity among Polish school- aged children and adolescents. Przegl Epidemiol. 2016;70(4):641-51.

7 Erdol S, Mazzucco W, Boccia S. Cost effectiveness analysis of childhood obesity primary prevention programmes: a systematic review. Epidemiol Biostat Public Heal. 2014 Jan;11(3).

8 Kovacs VA, Sliz D, Brzeziński M. Reducing childhood obesity in Poland by effective policies. 2017.

9 Brown T, Moore TH, Hooper L, Gao Y, Zayegh A, Ijaz S, et al. Do diet and physical activity strategies help prevent obesity in children (aged 0 to 18 years)? Cochrane Database Syst Rev. 2019;23:CD001871.

10 Kelishadi R, Azizi-Soleiman F. Controlling childhood obesity: A systematic review on strategies and challenges. J Res Med Sci. 2014 Oct;19(10):993-1008.

11 Zolotarjova J, Ten Velde G, Vreugdenhil AC. Effects of multidisciplinary interventions on weight loss and health outcomes in children and adolescents with morbid obesity. Obes Rev. 2018 Jul;19(7):931-46.

12 Szlagatys-Sidorkiewicz A, Brzeziński M, Jankowska A, Metelska P, Słomińska-Frączek M, Socha P. Long-term effects of vitamin D supplementation in vitamin D deficient obese children participating in an integrated weight-loss programme (a double-blind placebo-controlled study) - rationale for the study design. BMC Pediatr. 2017 Apr;17(1):97.

13 Brzezinski M, Jankowski M, Niedzielska A, Danielewicz A, Czarnecka P. Health program "6-10-14 for Health" as an example of comprehensive environmental activities in the field of children obesity: study protocol and primary results. Appetite. 2014;76:202.

14 Kułaga Z, Litwin M, Tkaczyk M, Palczewska I, Zajączkowska M, Zwolińska D, et al. Polish 2010 growth references for school-aged children and adolescents. Eur J Pediatr. 2011 May;170(5):599-609.

15 Bleich SN, Vercammen KA, Zatz LY, Frelier JM, Ebbeling CB, Peeters A. Interventions to prevent global childhood overweight and obesity: a systematic review. Lancet Diabetes Endocrinol. 2018 Apr;6(4):332-346.

16 Flodgren G, Gonçalves-Bradley DC, Summerbell CD. Interventions to change the behaviour of health professionals and the organisation of care to promote weight reduction in children and adults with overweight or obesity. Cochrane Database Syst Rev. 2017 Nov;11:CD000984.

17 Epstein LH, Paluch RA, Wrotniak BH, Daniel TO, Kilanowski C, Wilfley D, et al. Cost-effectiveness of familybased group treatment for child and parental obesity. Child Obes. 2014 Apr;10(2):114-21.

18 Janicke DM, Sallinen BJ, Perri MG, Lutes LD, Silverstein JH, Brumback B. Comparison of program costs for parent-only and family-based interventions for pediatric obesity in medically underserved rural settings. J Rural Health. 2009;25(3):326-30.

19 Quattrin T, Cao Y, Paluch RA, Roemmich JN, Ecker MA, Epstein LH. Cost-effectiveness of Family-Based Obesity Treatment. Pediatrics. 2017 Sep;140(3):e20162755.

20 Weinstein MC, Stason WB. Foundations of cost-effectiveness analysis for health and medical practices. N Engl J Med. 1977 Mar;296(13):716-21.

21 Su W, Huang J, Chen F, Iacobucci W, Mocarski M, Dall TM, et al. Modeling the clinical and economic implications of obesity using microsimulation. J Med Econ. 2015;18(11):886-97.

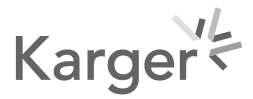


22 Oosterhoff M, Bosma H, van Schayck OC, Evers SM, Dirksen CD, Joore MA. A systematic review on economic evaluations of school-based lifestyle interventions targeting weight-related behaviours among 4-12 year olds: issues and ways forward. Prev Med. 2018 Sep;114:115-22.

23 Peirson L, Fitzpatrick-Lewis D, Morrison K, Ciliska D, Kenny M, Usman Ali M, et al. Prevention of overweight and obesity in children and youth: a systematic review and meta-analysis. CMAJ Open. 2015 Jan;3(1):E23-33.

24 Haby MM, Vos T, Carter R, Moodie M, Markwick A, Magnus A, et al. A new approach to assessing the health benefit from obesity interventions in children and adolescents: the assessing cost-effectiveness in obesity project. Int J Obes. 2006 Oct;30(10):1463-75.

25 Kropski JA, Keckley PH, Jensen GL. School-based obesity prevention programs: an evidence-based review. Obesity (Silver Spring). 2008 May;16(5):1009-18.

26 Moodie ML, Carter RC, Swinburn BA, Haby MM. The cost-effectiveness of Australia's active after-school communities program. Obesity (Silver Spring). 2010 Aug;18(8):1585-92.

27 Hung LS, Tidwell DK, Hall ME, Lee ML, Briley CA, Hunt BP. A meta-analysis of school-based obesity prevention programs demonstrates limited efficacy of decreasing childhood obesity. Nutr Res. 2015 Mar;35(3):229-40.

28 Brown EC, Buchan DS, Baker JS, Wyatt FB, Bocalini DS, Kilgore L. A Systematised Review of Primary School Whole Class Child Obesity Interventions: Effectiveness, Characteristics, and Strategies. BioMed Res Int. 2016; 2016:4902714.

29 Wang Y, Cai L, Wu Y, Wilson RF, Weston C, Fawole O, et al. What childhood obesity prevention programmes work? A systematic review and meta-analysis. Obes Rev. 2015 Jul;16(7):547-65.

30 Yang Y, Kang B, Lee EY, Yang HK, Kim HS, Lim SY, et al. Effect of an obesity prevention program focused on motivating environments in childhood: a school-based prospective study. Int J Obes. 2017 Jul;41(7):1027-34.

31 Robertson W, Fleming J, Kamal A, Hamborg T, Khan KA, Griffiths F, et al. Randomised controlled trial and economic evaluation of the 'Families for Health' programme to reduce obesity in children. Arch Dis Child. 2017 May;102(5):416-26.

32 Finkelstein EA, Graham WC, Malhotra R. Lifetime direct medical costs of childhood obesity. Pediatrics. 2014 May;133(5):854-62.

33 Hollingworth W, Hawkins J, Lawlor DA, Brown M, Marsh T, Kipping RR. Economic evaluation of lifestyle interventions to treat overweight or obesity in children. Int J Obes. 2012 Apr;36(4):559-66.

34 Wang LY, Gutin B, Barbeau P, Moore JB, Hanes J Jr, Johnson MH, et al. Cost-effectiveness of a school-based obesity prevention program [Erratum appears in J Sch Health. 2009 Feb;79(2):89]. J Sch Health. 2008 Dec; 78(12):619-24. [cited 2019 Oct 20].

35 Anderson YC, Leung W, Grant CC, Cave TL, Derraik JG, Cutfield WS, et al. Economic evaluation of a multi-disciplinary community-based intervention programme for New Zealand children and adolescents with obesity. Obes Res Clin Pract. 2018 May - Jun;12(3):293-8.

36 Al-Khudairy L, Loveman E, Colquitt JL, Mead E, Johnson RE, Fraser H, et al. Diet, physical activity and behavioural interventions for the treatment of overweight or obese adolescents aged 12 to 17 years. Cochrane Database Syst Rev. 2017 Jun;6(6):CD012691.

37 Brzeziński M, Korzeniowska K, Szarejko K, Radziwiłł M, Myśliwiec M, Anyszek T, et al. “PoZdro!” as an example of a successful multicenter programme for obesity management and healthy lifestyle promotion in children and adolescents - programme protocol and preliminary results from the first intervention site. Pediatr Endocrinol Diabetes Metab. 2020;26(1):22-6. 